\title{
Emergent Serum Therapy and Antibody Medicine to Counteract Sudden Attacks of COVID-19 and Other Pathogenic Epidemics
}

\author{
Peter K. Law \\ Cell Therapy Institute, Wuhan, China \\ Email: peter@celltherapy.com
}

How to cite this paper: Law. P.K. (2020) Emergent Serum Therapy and Antibody Medicine to Counteract Sudden Attacks of COVID-19 and Other Pathogenic Epidemics. Open Journal of Regenerative Medicine, 9, 1-7.

https://doi.org/10.4236/ojrm.2020.91001

Received: March 2, 2020

Accepted: March 3, 2020

Published: March 6, 2020

Copyright $\odot 2020$ by author(s) and Scientific Research Publishing Inc. This work is licensed under the Creative Commons Attribution International License (CC BY 4.0). http://creativecommons.org/licenses/by/4.0/

\begin{abstract}
The science behind two life-saving protocols to combat COVID-19 and future pathogenic epidemics is presented for immediate implementation.
\end{abstract}

\section{Keywords}

Serum Therapy, Plasma Therapy, COVID-19, Coronavirus, Vaccine, Antibody Medicine

Pandemic destruction of Mad Cow Disease, Ebola, MERS, SARS and COVID-19 surfaced all within the past three decades seemed to indicate that various pathogens, be it bacterial or viral, have been "mutating" at a supernaturally rapid pace, with higher potency each time, to conquer the world on enemy grounds. It reminds me of the viral vector (P-shuttle SN Vector) technology of an American gene therapy team headed by Dr. French Anderson in the mid 1990's who could inactivate viruses with DNA splicing just as easy as they could insert DNA or RNA in them with promotors and enhancers. For the greatest benefit to humankind, implementation of therapies and emergent protocols are long-overdue to save-guard human lives and public health of the world.

On February 28, 2020, the National Health Commission of the People's Republic of China (NHCPRC) announced that of the 157 critically-ill COVID-19 subjects transfused with processed plasma collected from rehabilitated patients, 91 showed significant improvement within 48 hours of treatment. Improvement included increases in lymphocyte ratio, blood oxygen saturation, and reduction in viral antigen level. In addition, there was significant symptomatic improve- 
ment such as reduction and absence of fever, coughs, sputum, muscle pain and weakness. The NHCPRC declared that plasma therapy was safe and efficacious. China had collected 544 samples of donor plasma from rehabilitated volunteer donors to treat 245 cases of critically-ill patients. NHCPRC is waiting for confirmatory results with additional data and statistical analyses. It is in a major catastrophe such as COVID-19 in China that a large number of donated plasma samples are available for blood type matching in the plasma therapy, and for identification and isolation of the maximum numbers of viral antigens and antidotal antibodies, thus enabling the manufacture of diagnostic kits and antibody vaccine for treatment.

Plasma or serum therapy usage can be traced to diphtheria combat by Emil Adolf von Behring who was the first Nobel laureate in Physiology or Medicine in the year 1901. However, with the advent of antibiotics and steroids, plasma therapy has never been recognized as a treatment modality. Rather, it was used only when everything failed to stop patients from dying, such as in the cases of SARS and COVID-19. Often it was administered to individuals or small groups of patients, thus lacking statistics to validate its efficacy. Inasmuch as it is scientifically valid, user-friendly, requiring no regulatory approval, and has produced inconsistent but "magically" safe and efficacious results on patients, its practice is still needing technical refinement. China is collecting additional supportive evidence to substantiate plasma therapy as a treatment modality of COVID-19. At very low costs, its practice should be administered by national organizations responsible for public health and human services, with precision, great care, and free to the needy citizens.

We must recognize that antibiotics and steroids are symptom relievers and are not the real antidote for the viruses and their antigens. Even if the virus is genotyped, and monoclonal antibodies produced against its RNA, such "vaccine" cannot get pass the virus capsule, and thus cannot destroy the virus RNA. The coronaviruses gain entry into the epithelial cells of the lung alveolae, the myocardium, and the alimentary system through binding of their S-spike glycoprotein with the ACE-2 receptor sites present on the surface membrane of these cells. Presumably, such binding alters the structural conformation of the cell membrane and allows the viruses to get inside the cells. Once inside the cell, the viral RNA utilizes the host ribosome, mitochondria, and even nuclear hardware and software to multiply and mutate, producing enormous quantity of unidentified antigens, and killing the cells. For an etiologically correct remedy, we need a fauna of antibodies, each antidotal to its causative viral antigen. Evolution of the placental mammals over the past 160 million years witnesses a built-in immune system capable of doing just that. This occurred in the sera of the rehabilitated patients who had successfully won over the etiologic and symptomatic challenges. It is based on the pathogenesis and immunology of COVID-19 that I designed two protocols to combat any current and future onsets of sudden pathogenic epidemic attacks. 
Serum and plasma are two different components of whole blood. Serum is plasma without fibrinogen, which can easily be removed upon blood clots. Fibrinogen is a blood clot factor having a normal concentration of $2-4 \mathrm{~g} / \mathrm{L}$ of whole blood. In elderly plasma donors recovered from the infectious disease of COVID-19, especially with previous history of heart disease, lung inflammation, or kidney failure, fibrinogen concentration can increase substantially to 8 to $12 \mathrm{~g}$ in the $400 \mathrm{~mL}$ of plasma transfusion administered to the critically-ill subject. Though exerting no graft versus host reaction, the large quantity of fibrinogen is nonetheless a foreign antigen that the host macrophages will engulf, with cytokines and lysozymes be produced against them. The large dose of fibrinogen in the plasma therapy composition therefore greatly reduces the immunologic potency of the macrophages and the T-cells against the viruses, considering that these cells have already been hampered by prolonged usage of high doses of antibiotics and steroids. For effective usage, fibrinogen of donor plasmas must be removed to avoid allergy and inconsistency in treatment effectiveness. This new form of therapy is called "serum therapy" to befit proper nomenclature.

Due to limited supply of donated plasma, plasma therapy was usually conducted without adequate type-matching and screening for HIV, HBV, HCV, syphilis, endotoxins, mycoplasma, and bacteria. These procedures must be implemented following standard operation procedures (SOPs) with quality assurance for the serum therapy protocol that I proposed for emergent treatment of the critically-ill and dying patients. Past and current practices of plasma therapy were far from adhering to these standards, thus accounting for inconsistence in efficacy, sometimes allergic reaction, and in extreme cases, death of the patients.

There has not been enough attention paid to remove macrophages, $\mathrm{T}$ cells and $\mathrm{B}$ cells from the donated plasma. Supposedly, they were returned to the donor's blood stream, but very often some remained in the donor plasma. Following transfusion, they initiated a host of graft-versus-host reactions that greatly compromised the immunologic capability of the host patients. Furthermore, in the process of eliminating traces of viruses, bacteria, donor macrophages and lymphocytes, the donated sera used in transfusion must not be overheated for fear of denaturizing the antibodies.

Death by coronavirus is often associated with acute respiratory distress syndrome (ARDS) triggered by storms of cytokine release of TNF-alpha, IL-1, IL-6, IL-8, IL-12, IFN-alpha, IFN-beta, IFN-gamma, and MCP-1 by macrophages and the lymphatic cells into the lymphatic system, killing host and viruses indiscriminately. The patient usually died of pneumonia having high fever and multiple organ failure, but neural function was relatively spared.

The fear of cytokine storms and ARDS has always been the excuse not to apply plasma therapy to treat critically-ill patients. It would be good if the patients were diagnosed early when there were less viral antigens qualitatively and quantitatively for the donor antibodies to cope with. It would be good if there were enough donor plasma to work with, but this is not often the case. When the patient progressed into being critically- or terminally-ill, their immune systems 
have already been compromised with prolonged use of high doses of antibiotics and steroids for reduction of inflammation and fever. Many of the macrophages had died, the remaining ones stayed sick, with their immunologic capability much reduced by antibiotics and steroids. The healthy macrophages had to engulf a high dose of fibrinogen, the B-cells were producing antibodies against the donor fibrinogen and the $\mathrm{T}$ cells were attacking the donor fibrinogen. All of them were at the mercy of the viruses that had proliferated and mutated with new vigor to take over the human body. Only the donor antibodies could put them in check. The sick macrophages and worn-out T cells simply "exploded", emitting a swan song amidst cytokine storms of ARDS. Therefore, proper use of antibiotics and steroids without exceeding recommended dosage and duration of use become a must to avoid suicidal lymphocytic cells, cytokine storms and ARDS.

All these technical improvements deem my serum therapy protocol far more scientific and superior to the current practice of plasma therapy. There is much room for improvement and serum therapy will yield better results in safety and efficacy than plasma therapy.

Technically perfected and properly administered, the serum therapy is a powerful life-saver indeed. It is capable of eliminating the viruses within 48 hours, saving the lives of terminally-ill patients at the brink of death. What happened to the donor antibodies that remained after the victory? After all, they were allografts that would provoke rejection. There were no foreign macrophages or lymphocytes that might initiate graft-versus-host reaction. With the host immune system barely turned stable, it began to develop immunotolerance towards these non-invasive proteins and polypeptides. This is when the steroids and antibiotics must be weaned off, to be replaced with medical doses of anti-oxidants such as Coenzyme Q-10, Vitamin E, Vitamin C, or Omega 3, 6.9 to neutralize remaining cytokines and lysozymes and to promote body cell regeneration in various damaged organs. Some Chinese herbal medicine is excellent in dilating the trachea and the bronchial to allow the sputum to be coughed up. When physically permitted, the rehabilitated patients should be trained on Qi Gong which provides a moderate form of pulmonary exercise and rehabilitation.

The death toll is subsiding. There is a rumor that it was the on-coming warm weather that killed the coronavirus. The rumor claimed that the virus would perish at temperature over $25^{\circ} \mathrm{C}$, ignoring the fact that the same viruses usually claimed the human body at $42^{\circ} \mathrm{C}$. It is the ultra-violet sun wave that kills the viruses. The loss of destructive potency came after the coronaviruses had proliferated though certain number of generations; the number still awaited to be determined. This is a direct evidence that the coronavirus responsible for COVID-19 was engineered or man-made. "Transduced" viruses always revert to their wild type, losing their insertions of transduced DNA that conveyed fatality to humans. This is analogous to a soldier like Rambo losing an arm or two after many battles overseas. In time, all "transduced" viruses will lose the killing capacity that they were engineered for, just like soldiers turning to become civilians. The 
common cold virus never disappears like the MERS and the SARS viruses because the cold viruses are natural products of evolution through tens of millions of years.

As of March 1, 2020, there are 41,675 rehabilitated patients and 35 critically-ill patients died the day before. There was enough donor plasma to save these patients at the fortnight before their death, if we could systematically identify them according to their statistics that reflected their physical condition using mega data analyses. More than $85 \%$ of the 79,968 patients diagnosed with COVID-19 using nucleic acid testing and computed tomography exhibited mild symptoms, that were often suppressed with steroids, antibiotics and Chinese herbal medicine. Some of these patients, usually young and in good health before contracting COVID-19, eventually developed antibodies and recovered on their own within a month. The elderly patients, especially those with inherent illnesses such as cardiomyopathies, type-II diabetes and cancer, deteriorated within two weeks to become critically-ill patients. It is this latter group of patients that China had set a priority to receive the plasma therapy now, until enough donated plasma is collected for every patient. Of course, everyone is waiting for the time-consuming development and compassionate usage of an effective vaccine or antibody medicine.

The death toll has accumulated to 2873, yielding an average death rate of $3.592 \%$ nation-wide, the latter being highest in Wuhan because of the long incubation period of the coronavirus, rapid spreading of the disease, and lack of isolation and diagnostic/treatment resources during the early period of infection. The death rate peaked on January 27 , four days after the city lockdown of $\mathrm{Wu}$ han, but has decreased from the $7.2 \%$ to $4 \%$ since then. The death toll has steadily been reducing, from 132 on January 29 to 35 on March 1, 2020. At this rate, death over COVID-19 will disappear or reduced to a bare minimum in China by the end of March, 2020. It is time for us to pass-on our knowledge and experience to our friends overseas.

The second protocol I proposed is more time consuming and should be conducted as soon as donor sera are available. It conveys the advantages of amplifying almost indefinitely the treatment capacity of serum transfusion therapy by identifying the antidotal antigens for the manufacture of preventive vaccine and antibody medicine, in addition to discovering viral antigens for early diagnosis. Its design is self-explanatory as depicted in Figure 1.

Serum samples of each group must be pooled to include as much antigens and as much antibodies for analyses, especially in the fourth group where more qualitatively different antibodies provide greater power to counteract the different antigens from mutation of the viruses.

Using mass spectrometry, isoelectric focusing, and high pressure liquid chromatography, we aim to identify, isolate and purify viral antigens for the preparation of ELISA kits for diagnosis, by comparing Group 1 versus Group 2 sera (Figure 2 and Figure 3). In addition, multiple monoclonal antibodies against the viral antigens will be produced to treat early-infected patients exhibiting 


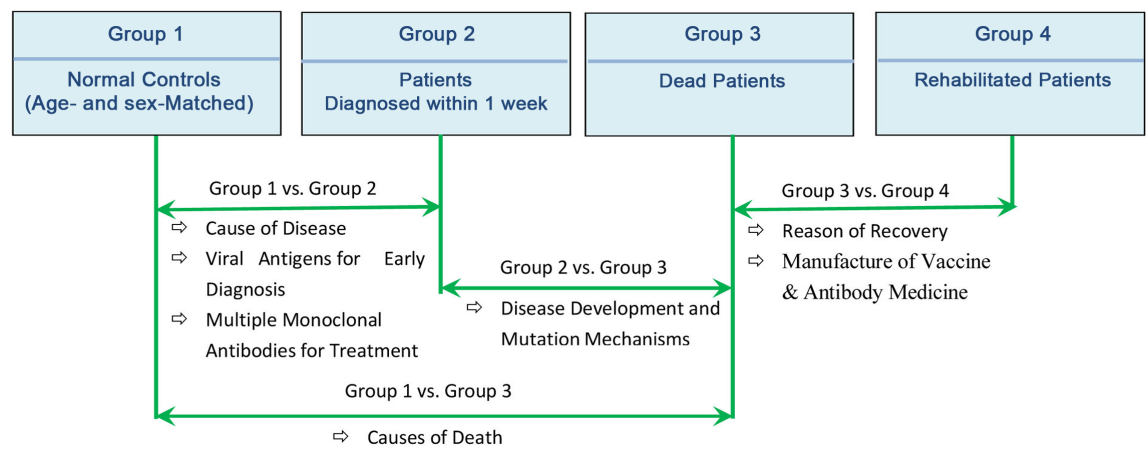

Figure 1. Schematic design for identification of viral antigens and effective antibodies by comparing the protein and polypeptide contents of the groups of sera donated from the four groups of subjects stated above.

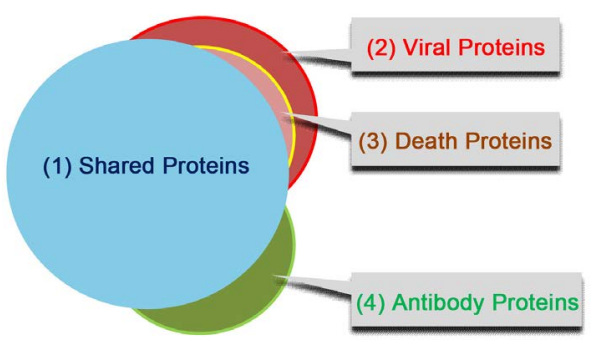

Figure 2. Hypothetic sets of protein contents from the comparison and qualitative analyses of the four serum groups depicted in Figure 1.
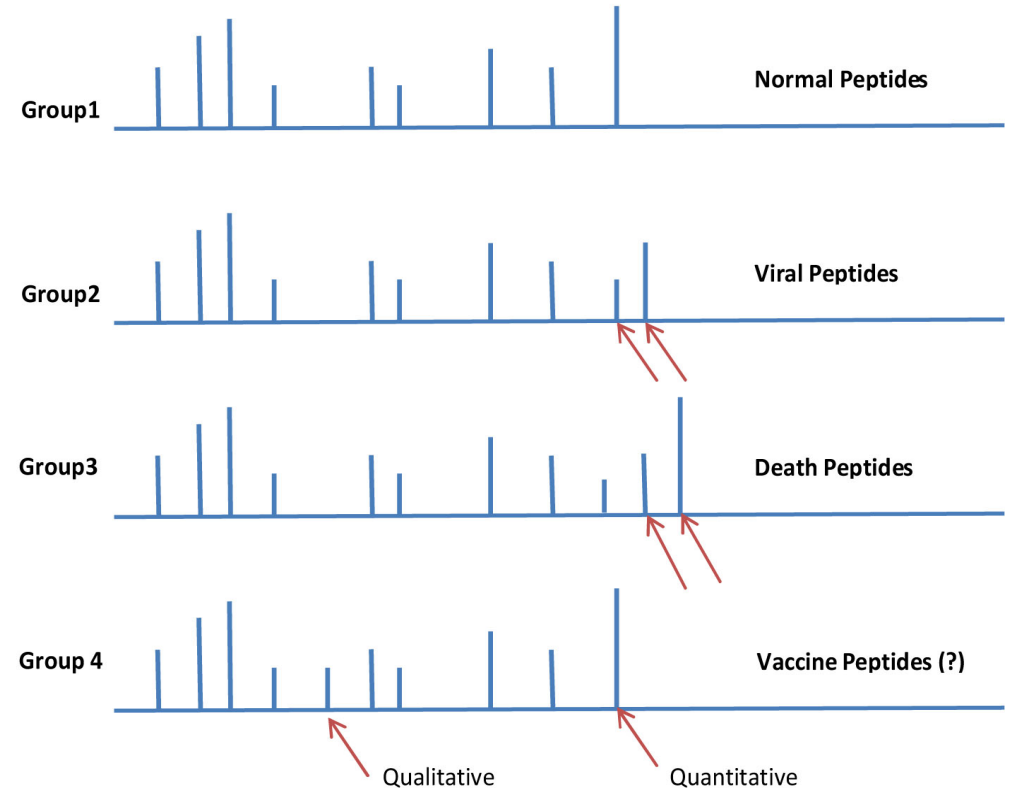

Figure 3. Hypothetic sets of polypeptide contents using high pressure liquid chromatography (HPLC) qualitative and quantitative analyses for the identification, isolation and purification of antigens and antibodies in the four groups of sera depicted in Figure 1.

mild symptoms. By comparing Group 3 versus Group 4 sera, we aim to identify, isolate and purify antidotal antibodies for mass-production of antibody medicine and vaccine to prevent infection (Figure 2 and Figure 3 ). 
I told the CDC in Atlanta in late 2002 when a CDC scientist called me for advices against SARS, but no one has followed through with any scientific publication. I submitted the antibody medicine protocol to Hubei Provincial Department of Science and Technology on January 27, and wrote to appraise its officials of the serum therapy on February 12. I had also submitted these protocols to the National Scientific and Technologic Research Group of the Joint Prevention and Control Mechanism of the State Council of China. However, the current practice of serum therapy in China is far from being perfect, and has to be "up-graded" to serum therapy according to what I proposed for greater safety and efficacy.

It is my hope that these urgently-needed information be disseminated throughout the globe, through scientific publications and news media, for interested parties to implement these protocols to counteract not only COVID-19, but all future sudden attacks of pathogenic epidemics. 